\title{
Maximizing antimalarial efficacy and the importance of dosing strategies
}

\author{
James G Beeson*, Philippe Boeuf and Freya Jl Fowkes
}

\begin{abstract}
Artemisinin-based combination therapies (ACTs) are the cornerstone for the treatment of malaria. However, confirmed resistance to artemisinins in South-East Asia, and reports of reduced efficacy of ACTs raise major concerns for malaria treatment and control. Without new drugs to replace artemisinins, it is essential to define dosing strategies that maximize therapeutic efficacy, limit the spread of resistance, and preserve the clinical value of ACTs. It is important to determine the extent to which reduced efficacy of ACTs reflects true resistance versus sub-optimal dosing, and quantify other factors that determine treatment failure. Pooled analyses of individual patient data from multiple clinical trials, by investigators in the Worldwide Antimalarial Resistance Network, have shown high overall efficacy for three widely used $\mathrm{ACTs}$, artemether-lumefantrine, artesunate-amodiaquine, and dihydroartemisinin-piperaquine. Analyses also highlight that suboptimal dosing leads to increased risk of treatment failure, especially among children. In the most recent study, an analysis of clinical trials of artesunate-amodiaquine, widely used among children in Africa, revealed a superior efficacy for fixed-dose combination tablets compared to loose non-fixed dose combinations. This highlights the benefits of fixed-dose combinations as a practical strategy for ensuring optimal antimalarial dosing and maximizing efficacy.

Please see related article: http://www.biomedcentral.com/1741-7015/13/66
\end{abstract}

Keywords: Amodiaquine, Artemisinins, Artesunate, Drug resistance, Malaria

\section{Introduction}

Artemisinin-based combination therapies (ACTs) have made a major contribution to the reduction in global malaria morbidity and mortality since their use became widespread approximately 10 years ago. ACTs are recommended by the WHO as the first-line treatment of uncomplicated and severe $P$. falciparum malaria in all areas in which malaria is endemic [1], and have been adopted as first-line therapy in many countries. Around 390 million ACT treatments are procured annually [2]. Drugs of the artemisinin group (artesunate, artemether, and dihydroartemisinin are the most used) are the most parasiticidal of established antimalarials and rapidly clear parasitemia, and are well tolerated with a good safety profile. However, artemisinin drugs have a short half-life ( $<1$ hour) and when used on their own to treat malaria require 7- to 10-day treatment to achieve high cure rates, which impacts on adherence. Therefore, artemisinins are

\footnotetext{
* Correspondence: beeson@burnet.edu.au

Burnet Institute, 85 Commercial Road, Melbourne, Victoria 3004, Australia
}

typically combined with a long-acting partner drug (e.g., lumefantrine, amodiaquine, piperaquine) in order to achieve high cure rates with a 3-day treatment regimen. The combination of artemether-lumefantrine is the most widely used ACT, and is highly efficacious [3]. Artesunateamodiaquine (AS-AQ) is widely used for malaria therapy in Africa, particularly among children, and is the second most used ACT globally.

\section{The global threat of drug resistance}

Unfortunately, the early signs of artemisinin resistance have emerged in South-East Asia threatening recent gains and milestones in the treatment and control of malaria $[4,5]$. Resistance to artemisinins has been recently associated with a mutation in the kelch13 gene (gene ID PF3D7_1343700) [6-8], and the identification of this genetic marker will greatly facilitate resistance surveillance $[4,9]$. Emerging resistance was initially identified as delayed parasite clearance rates following treatment with artemisinin-based therapies [5]. Confirmed partial artemisinin resistance is now defined by the 
WHO as $\geq 5 \%$ of patients carrying K13 resistanceassociated mutations, all of whom have been found, after treatment with ACT or artesunate monotherapy, to have either persistent parasitaemia by microscopy on day 3, or a parasite clearance half-life of $\geq 5$ hours. Reflecting the importance of this issue, the WHO launched its Global Plan on Artemisinin Resistance Containment in 2011 with a specific emergency response to artemisinin resistance in the Greater Mekong sub-region in 2013. In addition, there are reports of reduced clinical efficacy of ACT therapy after 28 days of follow-up in some settings [10-15]. It is important to determine the extent to which this reduced efficacy reflects true resistance versus suboptimal dosing, or other factors. The development of widespread resistance has limited the utility of numerous other antimalarials that were previously widely used, such as chloroquine and sulfadoxine-pyrimethamine, providing a sobering reminder of the potential impact of evolving resistance to drugs in current use. With no new drugs immediately available to replace artemisinins, it is essential to optimize and define dosing strategies to ensure maximum therapeutic efficacy of ACTs, limit the spread of resistance, and extend the clinical life of ACTs.

\section{Identifying factors associated with reduced efficacy: importance of dosing strategies}

A new study from the Worldwide Antimalarial Resistance Network (WWARN) investigated risk factors for treatment failure with AS-AQ therapy [16]. Reduced antimalarial efficacy of AS-AQ has been reported in some studies, but it is unlikely that drug resistance is the major factor explaining this; confirmed AS resistance has not yet been reported in Africa [4,17] and reduced efficacy of AS-AQ has been observed between studies within the same region (which should have similar rates of potential AQ resistance) [18-20]. It was hypothesised that differences in doses or formulations impacted on the antimalarial efficacy of AS-AQ. To investigate this, the WWARN investigator group conducted a systematic review and meta-analysis of individual patient data including published and unpublished antimalarial therapeutic efficacy trials that included at least one AS-AQ arm, conducted between 1999 and 2012.

AS-AQ is available in three different body-weightadjusted drug formulations: fixed-dose combinations, loose non-fixed dose combinations, and co-blistered non-fixed-dose combinations. All of these combinations aim at delivering $12 \mathrm{mg} / \mathrm{kg}$ of AS over 3 days, but the total target dose of AQ ranges from 25 to $30 \mathrm{mg} / \mathrm{kg}$ for loose non-fixed dose combinations and is $30 \mathrm{mg} / \mathrm{kg}$ for co-blistered non-fixed-dose combinations and fixed-dose combinations. The WWARN study included 43 trials (9,106 treatments), of which 39 trials (95\% of subjects) were conducted in Africa, as well as 3 in Asia and 1 in
South America, and the great majority were children (87.5\% were $<12$ years old) [16]. The authors investigated the relationship between these different drug formulations and the actual dose of AQ received as well as treatment success, including parasitemia at 28 days and potential recrudescence in infants, children, and adults. Studies of this size are needed in order to have sufficient power to examine these questions when treatment efficacy rates are high, as they are with ACTs, emphasising the importance of multisite collaborative studies.

Arguably, the most important finding was that fixeddose combinations were associated with the highest ASAQ treatment efficacy in all age groups, including in children less than 5 years of age, independently of high baseline parasitemia and young age. The fixed dose combination of AS-AQ was developed using weight-for-age values from malaria endemic countries, to ensure optimal dosing [21,22]. Loose non-fixed-dose combinations, with an AQ target dose of $25 \mathrm{mg} / \mathrm{kg}$, were associated with a 3.5-fold greater risk of recrudescence by day 28 . The implication of these findings on the treatment of paediatric malaria reinforces initiatives to promote the distribution and implementation of fixed-dose regimens for therapy. The use of co-blistered and loose non-fixeddose combinations may require splitting of tablets when treating children, potentially leading to suboptimal AQ dosing and therefore lower treatment efficacy. The use of fixed-dose AS-AQ formulations, including paediatric tablets, would circumvent this issue, leading to optimal AQ dosing and high treatment efficacy. As such, the treatment of uncomplicated P. falciparum malaria using fixed-dose AS-AQ formulations should be promoted for national treatment guidelines.

The study also found that the risk of recrudescence after AS-AQ treatment was higher among young children $(<12$ years old), those with a high baseline parasitemia, and in Asian studies compared to African. This may be reflective of the overall higher level of AQ resistance in Asia; however, given the small number of subjects from Asian trials, further studies are needed to examine this. The potentially crucial impact of P. falciparum resistance to AQ could not be evaluated in this study and further studies incorporating molecular markers of AQ resistance are warranted. Resistance to AQ is associated with mutations in the pfcrt and pfmdr genes [23,24], which are prevalent in most endemic countries [25]. It will be interesting to see the results from an AQ pharmacokinetic-pharmacodynamic analysis by WWARN, which is examining the effects of drug formulation and dose, and host age and nutritional status on AQ drug concentrations. Since most of the therapeutic efficacy trials analysed were performed in sub-Saharan Africa, more data from Asian and South American trials are needed in order to generalise these findings to 
different source populations with different risk factors and varied degrees of AQ resistance. Regarding side effects, there was no evidence for a higher risk of neutropenia (which is associated with AQ use) with the higher AQ dose, but higher rates of vomiting and diarrhoea were observed.

\section{Other studies investigating the importance of correct dosing with ACTs}

There have been two related studies performed by WWARN investigators on the efficacy of other ACTs, highlighting the importance of optimal dosing. Dihydroartemisinin-piperaquine is another widely used ACT, and risk factors for recrudescence after treatment were examined in a pooled analysis of individual patient data from 26 efficacy studies (7,072 patients) [26]. Overall efficacy was high $(97.7 \%)$, but was significantly reduced in those who received lower doses of piperaquine. Of concern was that $28.6 \%$ of young children (1 to 5 years old) received a piperaquine dose below the lower limit recommended by the WHO, further highlighting the need for strategies to ensure optimal dosing for malaria treatment. Recently, a pooled analysis of individual patient data from efficacy studies of artemetherlumefantrine included 61 studies and 14,327 patients [3]; overall, day 28 efficacy was very high (97.6\%). Analysis revealed that a higher dose of artemether was associated with a lower risk of persistent parasitaemia on day 1 and lower gametocyte carriage rates, which may be important for reducing transmission. In Asia, lower doses of lumefantrine were associated with reduced efficacy among children weighing 10 to $15 \mathrm{~kg}$. The risk of treatment failure was also higher among malnourished children aged 1 to 3 years in Africa.

\section{Conclusions}

In an era of emerging artemisinin resistance, and the push for malaria elimination in many regions, studies such as that of the WWARN collaboration provide important data to inform policymakers and clinicians to optimise antimalarial therapies to maximize efficacy and help reduce the evolution of resistance (see Box 1 for a summary of key points). These findings provide further support for international collaborative networks and data sharing arrangements to address major challenges in global health, and the approach used for malaria has strong relevance to antimicrobial therapy and resistance more broadly. Individual patient meta-analyses of therapeutic efficacy studies are considered the strongest form of clinical evidence, and are essential to inform antimalarial policy and clinical treatment guidelines to ensure the rapid and effective treatment of malaria cases.

\section{Box 1: Key points}

Antimalarial drugs and resistance

- The WHO recommends artemisinin combination therapies (ACT) as the first-line treatment of malaria

- Around 390 million courses of ACTs procured annually ${ }^{1}$

- Emerging resistance to ACTs: Resistance confirmed in five countries in the Greater Mekong sub-region

- Suspected artemisinin resistance is defined as failure to clear parasitemia within 3 days of commencing treatment, or a parasite clearance half-life of $\geq 5$ hours

- Resistance to ACTs is associated with mutations in kelch13 gene

- Resistance to other antimalarials is widespread (e.g. chloroquine, amodiaquine, sulfadoxine-pyrimethamine)

- Resistance to amodiaquine and chloroquine is associated with mutations in the pfcrt and pfmdrl genes

- Ensuring effective dosing is crucial to maximize efficacy and to protect against the further development and spread of resistance

\section{WWARN study on artesunate-amodiaquine (AS-AQ) ${ }^{2}$}

- AS-AQ is widely used in Africa, and is the second most widely used ACT

- AS-AQ Previously shown to have high efficacy when administered correctly

- WWARN study performed an individual patient metaanalysis using data from 43 studies (39 studies in Africa) with over 9,000 treatments, predominantly in children

- Study evaluated the impact of different dosing strategies on therapeutic efficacy

- Fixed-dose and co-blistered non-fixed dose combinations had high efficacy (98\%)

- Use of loose tablets at non-fixed dose had significantly lower efficacy (up to 3.5-fold higher risk of treatment failure)

- Findings highlight the benefit of fixed dose combinations and support this strategy in the treatment and control of malaria

- Increased risk of recrudescence among young children, those with high baseline parasitemia, and among trials conducted in Asia

1. Based on WHO figures from 2013.

2. See reference [16] 


\section{Abbreviations}

ACTs: Artemisinin-based combination therapies; AS-AQ: Artesunateamodiaquine; WWARN: Worldwide Antimalarial Resistance Network.

\section{Competing interests}

The authors declare that they have no competing interests.

\section{Authors' contributions}

All authors contributed equally to the preparation of this manuscript, and all authors approved the final version.

\section{Authors' information}

JGB is a malaria research group leader and public health physician at the Burnet Institute, an independent non-profit organisation, and a Senior Research Fellow of the National Health and Medical Research Council. PB is a senior research officer at the Burnet Institute, with a focus on malaria in pregnancy and maternal and fetal health. FJIF is malaria research group leader and infectious disease epidemiologist at the Burnet Institute, and a Future Fellow of the Australia Research Council.

\section{Acknowledgements}

Funding was provided by the National Health and Medical Research Council of Australia (Senior Research Fellowship and Project Grant to JGB) and the Australia Research Council (Future Fellowship to FJIF). The Burnet Institute is supported by the NHMRC Independent Research Institutes Infrastructure Support Scheme, and a Victoria State Government Operational Infrastructure Support grant. Thanks to Ric Price for helpful discussions.

Received: 16 April 2015 Accepted: 16 April 2015

Published online: 09 May 2015

\section{References}

1. World Health Organisation. Guidelines for the treatment of malaria. 2nd ed. Geneva: World Health Organisation; 2010.

2. World Health Organisation. World malaria report 2014. Geneva: World Health Organisation; 2014.

3. Worldwide Antimalarial Resistance Network ALDISG. The effect of dose on the antimalarial efficacy of artemether-lumefantrine: a systematic review and pooled analysis of individual patient data. Lancet Infect Dis. 2015. Ahead of print.

4. Ashley EA, Dhorda M, Fairhurst RM, Amaratunga C, Lim P, Suon S, et al. Spread of artemisinin resistance in Plasmodium falciparum malaria. N Engl J Med. 2014;371:411-23.

5. Dondorp AM, Nosten F, Yi P, Das D, Phyo AP, Tarning J, et al. Artemisinin resistance in Plasmodium falciparum malaria. N Engl J Med. 2009;361:455-67.

6. Ariey F, Witkowski B, Amaratunga C, Beghain J, Langlois AC, Khim N, et al. A molecular marker of artemisinin-resistant Plasmodium falciparum malaria. Nature. 2014;505:50-5

7. Straimer J, Gnadig NF, Witkowski B, Amaratunga C, Duru V, Ramadani AP et al. Drug resistance. K13-propeller mutations confer artemisinin resistance in Plasmodium falciparum clinical isolates. Science. 2015:347:428-31.

8. Mok S, Ashley EA, Ferreira PE, Zhu L, Lin Z, Yeo T, et al. Drug resistance. Population transcriptomics of human malaria parasites reveals the mechanism of artemisinin resistance. Science. 2015;347:431-5.

9. Tun KM, Imwong M, Lwin KM, Win AA, Hlaing TM, Hlaing $T$, et al. Spread of artemisinin-resistant Plasmodium falciparum in Myanmar: a cross-sectional survey of the K13 molecular marker. Lancet Infect Dis. 2015:15:415-21.

10. Rwagacondo CE, Karema C, Mugisha V, Erhart A, Dujardin JC, Van Overmeir $C_{\text {, et }}$ al. Is amodiaquine failing in Rwanda? Efficacy of amodiaquine alone and combined with artesunate in children with uncomplicated malaria. Trop Med Int Health. 2004;9:1091-8.

11. Bonnet M, Broek I, van Herp M, Urrutia PP, van Overmeir C, Kyomuhendo J, et al. Varying efficacy of artesunate + amodiaquine and artesunate + sulphadoxine-pyrimethamine for the treatment of uncomplicated falciparum malaria in the Democratic Republic of Congo: a report of two in-vivo studies. Malar J. 2009:8:192.

12. Mutabingwa TK, Anthony D, Heller A, Hallett R, Ahmed J, Drakeley C, et al. Amodiaquine alone, amodiaquine + sulfadoxine-pyrimethamine, amodiaquine + artesunate, and artemether-lumefantrine for outpatient treatment of malaria in Tanzanian children: a four-arm randomised effectiveness trial. Lancet. 2005;365:1474-80.
13. Adjuik M, Agnamey P, Babiker A, Borrmann S, Brasseur P, Cisse M, et al. Amodiaquine-artesunate versus amodiaquine for uncomplicated Plasmodium falciparum malaria in African children: a randomised, multicentre trial. Lancet. 2002;359:1365-72.

14. Smithuis F, Kyaw MK, Phe O, Win T, Aung PP, Oo AP, et al. Effectiveness of five artemisinin combination regimens with or without primaquine in uncomplicated falciparum malaria: an open-label randomised trial. Lancet Infect Dis. 2010;10:673-81.

15. Hasugian AR, Purba HL, Kenangalem E, Wuwung RM, Ebsworth EP, Maristela $R$, et al. Dihydroartemisinin-piperaquine versus artesunate-amodiaquine: superior efficacy and posttreatment prophylaxis against multidrug-resistant Plasmodium falciparum and Plasmodium vivax malaria. Clin Infect Dis. 2007:44:1067-74.

16. Worldwide Antimalarial Resistance Network AS-AQ Study Group. The effect of dosing strategies on the therapeutic efficacy of artesunate-amodiaquine for uncomplicated malaria: a meta-analysis of individual patient data. BMC Med. 2015;13:66.

17. Taylor SM, Parobek CM, DeConti DK, Kayentao K, Coulibaly SO, Greenwood $\mathrm{BM}$, et al. Absence of putative artemisinin resistance mutations among Plasmodium falciparum in Sub-Saharan Africa: a molecular epidemiologic study. J Infect Dis. 2015;211:680-8.

18. Zwang J, Olliaro P, Barennes $H$, Bonnet $M$, Brasseur $P$, Bukirwa $H$, et al. Efficacy of artesunate-amodiaquine for treating uncomplicated falciparum malaria in sub-Saharan Africa: a multi-centre analysis. Malar J. 2009:8:203.

19. Four Artemisinin-Based Combinations ( $4 A B C$ ) Study Group. A head-to-head comparison of four artemisinin-based combinations for treating uncomplicated malaria in African children: a randomized trial. PLoS Med. 2011;8:e1001119.

20. Thanh NX, Trung TN, Phong NC, Quang HH, Dai B, Shanks GD, et al. The efficacy and tolerability of artemisinin-piperaquine (Artequick(R)) versus artesunate-amodiaquine (Coarsucam) for the treatment of uncomplicated Plasmodium falciparum malaria in south-central Vietnam. Malar J. 2012;11:217

21. Sirima SB, Tiono AB, Gansane A, Diarra A, Ouedraogo A, Konate AT, et al. The efficacy and safety of a new fixed-dose combination of amodiaquine and artesunate in young African children with acute uncomplicated Plasmodium falciparum. Malar J. 2009:8:48.

22. Brasseur P, Agnamey P, Gaye $O$, Cisse M, Badiane M, Vaillant M, et al. Dosing accuracy of artesunate and amodiaquine as treatment for falciparum malaria in Casamance, Senegal. Trop Med Int Health. 2009;14:79-87.

23. Djimde A, Doumbo OK, Cortese JF, Kayentao K, Doumbo S, Diourte Y, et al. A molecular marker for chloroquine-resistant falciparum malaria. N Engl J Med. 2001:344:257-63.

24. Sidhu AB, Verdier-Pinard D, Fidock DA. Chloroquine resistance in Plasmodium falciparum malaria parasites conferred by pfcrt mutations. Science. 2002;298:210-3.

25. Venkatesan M, Gadalla NB, Stepniewska K, Dahal P, Nsanzabana C, Moriera $C$, et al. Polymorphisms in Plasmodium falciparum chloroquine resistance transporter and multidrug resistance 1 genes: parasite risk factors that affect treatment outcomes for $\mathrm{P}$. falciparum malaria after artemether-lumefantrine and artesunate-amodiaquine. Am J Trop Med Hyg. 2014;91:833-43.

26. Worldwide Antimalarial Resistance Network DP Study Group. The effect o $f$ dosing regimens on the antimalarial efficacy of dihydroartemisininpiperaquine: a pooled analysis of individual patient data. PLOS Med. 2013;10:e1001564; discussion e1001564.

\section{Submit your next manuscript to BioMed Central and take full advantage of:}

- Convenient online submission

- Thorough peer review

- No space constraints or color figure charges

- Immediate publication on acceptance

- Inclusion in PubMed, CAS, Scopus and Google Scholar

- Research which is freely available for redistribution 